\title{
Uso de la colaboración y la gamificación en MOOC: un análisis exploratorio
}

\section{The use of collaboration and gamification in MOOCs: an exploratory analysis}

\author{
Sara García-Sastre \\ Miriam Idrissi-Cao \\ Alejandro Ortega-Arranz \\ Eduardo Gómez-Sánchez \\ Universidad de Valladolid, UVa (España)
}

\section{Resumen}

Los cursos online masivos y abiertos (MOOC: Massive Open Online Courses) presentan un gran potencial para permitir el acceso a la educación superior a cualquier estudiante de manera gratuita y que tenga la posibilidad de acceder a Internet. Sin embargo, su modelo de enseñanza es habitualmente criticado, y múltiples investigadores defienden la aplicación de pedagogías activas para tratar de alcanzar un mejor aprendizaje, como ya es frecuente en el aula presencial. Las diferencias de contexto en estos cursos, como la heterogeneidad de perfiles, la escala o el asincronismo se argumentan con frecuencia como limitadores. Este artículo presenta la exploración de veinte cursos masivos, en diferentes idiomas, en distintas plataformas y en diversos dominios de conocimiento, con el objetivo de analizar en detalle el uso del aprendizaje colaborativo y de la gamificación en los mencionados cursos. Las evidencias encontradas permiten concluir que, a pesar de que hay una preocupación por fomentar la interacción social, no existe una intención pedagógica en ella; y además, si se produce el aprendizaje colaborativo es de manera espontánea, no buscada como el eje central del proceso en el diseño instruccional. Por otro lado, la gamificación sí está presente como elemento motivador, pero está dirigida fundamentalmente a premiar acciones individuales de los estudiantes, no los logros colectivos. También se observa que las distintas plataformas MOOC frecuentan ser complementadas con otras herramientas para tratar de apoyar la interacción de los participantes y la gamificación.

Palabras clave: cursos online; conceptualización; colaboración; motivación; gamificación.

\begin{abstract}
Massive Open Online Courses (MOOCs) have shown great potential to provide open access to learning, especially in higher education, with just Internet connection. However, the pedagogical model used in these courses is frequently being criticized. Many researchers claim the application of active pedagogies in MOOCs in order to improve the student learning processes, as it has been shown in face-to-face courses. Nevertheless, some common features
\end{abstract}


of MOOCs such as the heterogeneity of user profiles, the scale or the asynchronism can hinder the application of these active pedagogies. This paper explores twenty MOOCs, in different languages, platforms and knowledge domains to analyze in detail the current use of collaboration and gamification in such courses. Results show that although there is a trend to promote social interaction, there is a lack of underpinning pedagogical intentions to achieve collaborative outcomes, and when collaboration happens, it is mostly spontaneously, not pursued by the instructional design. Also, results show that gamification is frequently used as a motivational strategy, implemented for rewarding students' individual actions, lacking the recognition of collective achievements. Finally, there is evidence that MOOC platforms are often complemented with additional tools to support student interaction and gamification.

Keywords: online courses; conceptualization; collaboration; motivation; gamification.

Los avances en las Tecnologías de la Información y la Comunicación han revolucionado la manera en la que se accede al conocimiento y en la que es posible aprender y ofrecer formación. Por una parte, los recursos educativos abiertos permiten ensamblar con relativa facilidad diseños instruccionales basados en contenidos muy revisados y de alta calidad.

Por otra parte, las plataformas de enseñanza masiva permiten alcanzar a cientos de miles de personas.

En efecto, los MOOC se han vuelto muy populares, con miles de cursos producidos anualmente (Shah, 2015, 2016), a lo que se añade un gran impacto mediático que con frecuencia hace alusión a sus múltiples ventajas, como la democratización del acceso al conocimiento y el posible aprendizaje derivado de un contexto social masivo (Dillenbourg, Fox, Kirchner, Mitchell y Wirsing, 2014). A pesar de estas ventajas, la comunidad investigadora ha detectado una serie de limitaciones que este tipo de cursos presentan.

Uno de los principales problemas asociados a los MOOC es la motivación e implicación de los participantes que se matriculan en ellos. De manera cuantitativa, son muchos los matriculados en estos cursos, pero, sin embargo, la tasa de abandono también es muy elevada (Alario-Hoyos, Pérez-Sanagustín, Delgado-Kloos, ParadaGélvez, y Muñoz-Organero, 2014; Gütl, Rizzardini, Chang y Morales, 2014; Jordan, 2014; Poy y Gonzales-Aguilar, 2015). Algunos investigadores apuntan a la escasa presencia de pedagogías activas en tales cursos (Balula, 2015; Ferguson y Sharples, 2014; Fidalgo, Sein-Echaluce y García-Peñalvo, 2013), posiblemente debido a la dificultad de implementar sus diseños instruccionales, como causas del elevado abandono, por lo que su introducción se ve necesaria para mejorar los procesos de enseñanza-aprendizaje en este tipo de cursos (Poy y Gonzales-Aguilar, 2015).

Bonwell y Eison (1991) propusieron una lista de las estrategias más relevantes para promover un aprendizaje activo entre las que se encuentran la colaboración y el uso de juegos, actualmente transferible al contexto de gamificación. En lo que respecta al aprendizaje colaborativo, sus beneficios para mejorar la construcción de aprendizaje de manera social son ampliamente conocidos en entornos no masivos 
(Dillenbourg, 1999). Algunos autores como Claros, Echeverría, Garmendía y Cobos (2014), Zheng, Rosson, Shih y Carroll (2015) y Muñoz, Collazos y González (2016) presentan diferentes propuestas para incluir la colaboración en los MOOC. Por ejemplo, en Claros et al. (2014) se propone un modelo pedagógico para fomentar la colaboración en cursos masivos compuestos por distintos elementos (temas, escenarios de aprendizaje colaborativo, actividades y evaluación).

Por su parte, la gamificación también se ha propuesto con diferentes fines en este tipo de cursos masivos, tales como mejorar los resultados de aprendizaje, aumentar la retención de los estudiantes o fomentar su interacción. La gamificación es definida como el uso de elementos y estructuras típicas de los juegos (como medallas, misiones, narrativa) en contextos no lúdicos (De Sousa Borges, Durelli, Reis y Isotani, 2013; Deterding, Dixon, Khaled y Nacke, 2011), mostrando efectos positivos para los estudiantes dependiendo en gran medida de su contexto y diseño (Dichev y Dicheva, 2017; Dicheva, Dichev, Agre y Angelova, 2015; Hamari, Koivisto y Sarsa, 2014). Por ejemplo, para estudiar la influencia del diseño, Zeng (2016) estudia el proceso de diseño de un MOOC gamificado a través de varios factores funcionales, como la disposición del escenario de juego, el diseño del paso de nivel, las reglas del juego y el control de la dificultad.

La colaboración y la gamificación pueden combinarse como un recurso de gran utilidad para incrementar la motivación y la vinculación de los participantes hacia los MOOC en los que se registran, intentando reducir las tasas de abandono que en la actualidad son tan elevadas y, sobre todo, mejorar la calidad de su aprendizaje. Así, Muñoz et al. (2016) plantean en un MOOC el fomento de ambas pedagogías a través de técnicas y procesos relevantes para el diseño de sistemas gamificados y la inclusión de aspectos colaborativos. Para ello, estos autores presentan una propuesta para la gamificación de actividades colaborativas en entornos específicos de estos cursos.

Sin embargo, a pesar de los estudios mencionados, el uso de la colaboración (Manathunga y Hernández-Leo, 2015; Margaryan, Bianco y Littlejohn, 2015) y la gamificación (Dicheva et al., 2015; Ortega-Arranz, Muñoz-Cristóbal, MartínezMonés, Bote-Lorenzo y Asensio-Pérez, 2017a) es todavía minoritario en los MOOC, posiblemente a causa de limitaciones tecnológicas en las plataformas que los ofrecen.

En resumen, por una parte ya ha sido contrastado en pequeñas escalas que la colaboración y la gamificación presenta ventajas pedagógicas que podrían ser adecuadas para abordar algunos de los problemas pedagógicos presentes en los MOOC. Por otra, hay limitaciones en su aplicación, relacionadas con la tecnología, la heterogeneidad de perfiles del alumnado y las dificultades intrínsecas de escalar diseños educativos muy participativos. Por último, hay algunos intentos, no excesivamente numerosos, de aplicación de estas estrategias pedagógicas en este tipo de cursos. En este contexto, podría ser útil para los educadores y diseñadores instruccionales que deseen introducir aprendizaje colaborativo y/o gamificado en cursos masivos, conocer las experiencias anteriores, y aprender de los éxitos y los fracasos producidos. 
Por todo lo expuesto anteriormente, este artículo realiza un análisis exploratorio de cursos MOOC de diversas características analizando los distintos usos de la colaboración y la gamificación, sus intenciones pedagógicas y, cuando sea posible, las dificultades y los logros alcanzados. Para ello, se parte de un trabajo previo (GarcíaSastre, Idrissi-Cao, Ortega-Arranz, Muñoz-Cristóbal y Gómez-Sánchez, 2017) en el que se formula un marco de análisis de estas características para comprender cómo se plantean en los distintos MOOC la colaboración y la gamificación. Posteriormente se seleccionan una serie de cursos representativos, se discute este proceso, y se aplica el marco de análisis extrayendo una serie de conclusiones que puedan ser útiles para quien pretenda diseñar un MOOC colaborativo y/o gamificado.

El resto del artículo se estructura de la siguiente manera: la sección 2 recuerda las características principales del marco de análisis y describe el proceso de selección de cursos; la sección 3 presenta los resultados del análisis; finalmente, la última sección discute estos resultados y presenta las conclusiones incluyendo posibles líneas futuras de trabajo a seguir.

\section{MÉTODO}

Para afrontar este proceso de investigación se partió del trabajo previo realizado que presenta un marco de análisis (García-Sastre et al., 2017), formalizado en una serie de categorías relevantes propuestas a partir de la literatura y de la evaluación previa de algunos MOOC, con el objeto de sistematizar el grado de presencia del aprendizaje colaborativo y/o gamificado existente en los MOOC. El resultado se organizó en cinco categorías, algunas con subcategorías (véase figura 1.): A. Datos básicos; B. Mecanismos para favorecer la interacción y la comunicación entre participantes (creación de grupos, subgrupos, herramientas para intercambiar ideas y opiniones: foros, chats, redes sociales, etc.); C. Aprendizaje colaborativo (si está presente en el MOOC a través de la motivación, escala en la que se presenta, papel de la colaboración en el aprendizaje, recursos, evaluación colaborativa); D. Gamificación (si está presente en el MOOC con el fin que se utiliza, las mecánicas y dinámicas que se llevan a cabo, qué tipos de acciones y herramientas gamificadas); y, E. Observaciones.

El proceso investigador de generación del marco está descrito en (García-Sastre et al., 2017), pero adicionalmente el formulario de investigación generado por este marco en el que se listan con detalle cada uno de los elementos de análisis se recoge en el material complementario ${ }^{1}$ del presente artículo. 
Figura 1. Marco de análisis y categorías propuestas

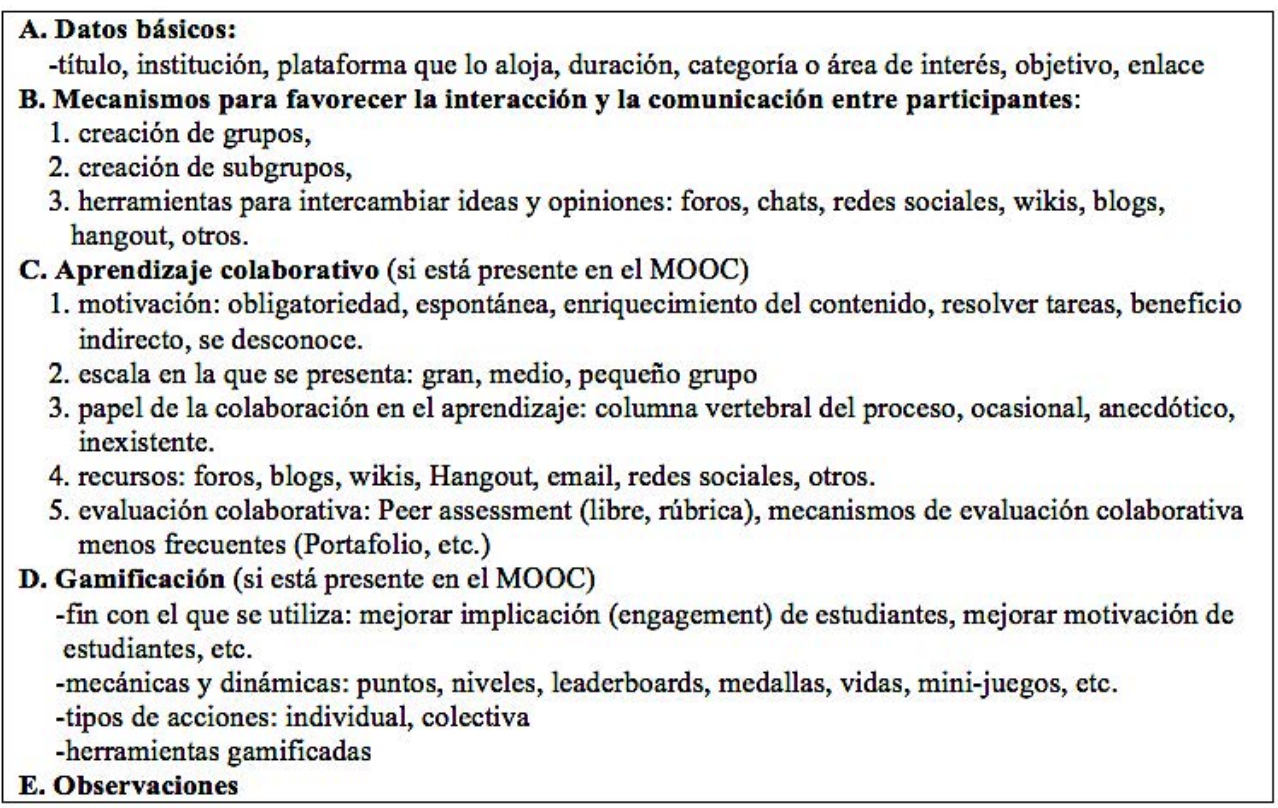

Fuente: Elaboración propia (2017)

En su conjunto, esta investigación se realizó en dos fases. Inicialmente, se llevó a cabo una revisión y análisis de la literatura para ahondar en las investigaciones actuales sobre el tipo de actividades en las que se fomenta la gamificación y/o colaboración diseñadas y puestas en marcha en los MOOC. Este análisis se realizó de manera individual por parte de tres investigadores, contrastando los hallazgos y poniendo en común la información seleccionada. En la segunda fase se observaron y analizaron veinte MOOC, para que de manera ilustrativa y representativa se pudieran valorar distintas cuestiones en relación con la colaboración y la gamificación.

La selección de estos MOOC comenzó por unas búsquedas realizadas por palabras clave ("gamificación", "colaboración” o "aprendizaje colaborativo", tanto en idioma español como en inglés) en el propio motor de búsqueda de las plataformas más populares que ofrecen MOOC, tales como Canvas Network ${ }^{2}$, ECOLearning (OpenMOOC) ${ }^{3}$, Coursera ${ }^{4}$, FutureLearn ${ }^{5}$, EdX ${ }^{6}, \mathrm{NovoEd}^{7}$, y MiríadaX $^{8}$, y un agregador de búsquedas llamado "class-central.com” ${ }^{9}$. Tras obtener los resultados, los investigadores procedieron a una inspección superficial de la información disponible en los listados de MOOC, y se seleccionaron algunos que pudieran ilustrar limitaciones tecnológicas de las plataformas en relación con la colaboración o la gamificación (Collazos, González y García, 2014) y otros que mostrasen el uso de 
ciertos elementos de apoyo a la colaboración (Ramírez-Donoso, Pérez-Sanagustín, Neyem y Rojas-Riethmuller, 2015) y a la gamificación (Hansch, Newman y Schildhauer, 2015). En esta selección se priorizaron la elección de cursos recientes, que pudieran reflejar aproximaciones actuales al diseño de cursos masivos.

La selección realizada de MOOC se limitó a veinte cursos por motivos de eficiencia y como representación de un número significativo de cursos. De modo que se procedió a la aplicación del marco de análisis por parte de dos investigadoras del equipo, recuperando en detalle toda la información posible de los aspectos colaborativos y gamificados en los distintos MOOC. Finalmente, el resultado del análisis se elaboró en un panel entre todo el equipo de investigación del presente trabajo. Para dar muestra de la diversidad de los cursos elegidos (tabla 1), cabe mencionar que hay quince cursos en inglés y cinco en español, ofrecidos por veinte instituciones distintas (como Universidad de Pensilvania, Indiana, Leeds, Gerona, Valladolid, Londres, Illinois, San Diego, Queensland o Michigan, entre otras), en las que algunas instituciones ofrecen el curso MOOC de manera conjunta (Universidad de Cantabria/Universidad de Oviedo/UNED). Cabe destacar la multiplicidad de plataformas que existen en la actualidad en las que se alojan estos cursos seleccionados (como Canvas Network ${ }^{2}$, EdX ${ }^{3}$, Coursera ${ }^{4}$, NovoEd ${ }^{5}$, FutureLearn ${ }^{6}$, OpenMOOC, MiríadaX $^{8}$ ), todas representadas en los cursos seleccionados. Los contenidos de los cursos abarcan distintas categorías o áreas de interés como: ciencias sociales, ciencias de la educación, diseño de cursos, desarrollo personal, etc.

\section{RESULTADOS}

La aplicación del marco de análisis a los veinte cursos seleccionados dio lugar a una ficha con información detallada de cada uno de ellos. En la tabla 1 se muestran los datos básicos (categoría A) más relevantes de los veinte cursos, asignando un identificador a cada uno de ellos. En las tablas 2, 3 y 4 se recogen los elementos más interesantes para la discusión (categorías $\mathrm{B}, \mathrm{C}$ y D, respectivamente) de cada uno de estos veinte cursos. Los detalles completos del análisis se proporcionan como material complementario ${ }^{1}$ a este artículo.

Tabla 1. Datos básicos más importantes de los 20 cursos analizados

\begin{tabular}{|c|c|c|c|c|c|}
\hline Id & \multicolumn{1}{|c|}{ Título } & Institución & Plataforma & Duración & $\begin{array}{c}\text { Área de } \\
\text { interés }\end{array}$ \\
\hline o1 & $\begin{array}{l}\text { Instructional Design } \\
\text { Service Course: Gain } \\
\text { Experience for Good }\end{array}$ & U. Illinois & Canvas Network & 12 semanas & $\begin{array}{l}\text { Diseño de } \\
\text { cursos }\end{array}$ \\
\hline
\end{tabular}




\begin{tabular}{|c|c|c|c|c|c|}
\hline Id & Título & Institución & Plataforma & Duración & $\begin{array}{l}\text { Área de } \\
\text { interés }\end{array}$ \\
\hline $\mathbf{0 2}$ & $\begin{array}{l}\text { Aprendiendo a } \\
\text { aprender: poderosas } \\
\text { herramientas mentales } \\
\text { con las que podrás } \\
\text { dominar temas difíciles } \\
\text { (Learning How to } \\
\text { Learn) }\end{array}$ & $\begin{array}{l}\text { U. California } \\
\text { San Diego }\end{array}$ & Coursera & 4 semanas & $\begin{array}{l}\text { Desarrollo } \\
\text { personal }\end{array}$ \\
\hline $\mathbf{0 3}$ & $\begin{array}{l}\text { Investigación científica } \\
\text { 2.0.1.: Procesos clave } \\
\text { en una sociedad digital } \\
\text { (2ed) }\end{array}$ & U. Girona & MiríadaX & 6 semanas & $\begin{array}{l}\text { Ciencia y } \\
\text { Tecnología }\end{array}$ \\
\hline 04 & $\begin{array}{l}\text { Innovación Educativa y } \\
\text { Desarrollo Profesional. } \\
\text { Posibilidades y límites } \\
\text { de las TIC. (5ed) }\end{array}$ & $\begin{array}{l}\text { U. Cantabria, } \\
\text { U. Oviedo, } \\
\text { UNED }\end{array}$ & OpenMOOC & 8 semanas & C. Educación \\
\hline $\mathbf{0 5}$ & $\begin{array}{l}\text { Alfabetización Digital } \\
\text { para Personas en } \\
\text { Riesgo de Exclusión: } \\
\text { Estrategias para } \\
\text { la Intervención } \\
\text { Socioeducativa. (3ed) }\end{array}$ & $\begin{array}{l}\text { U. Cantabria, } \\
\text { U. Oviedo, } \\
\text { UNED }\end{array}$ & OpenMOOC & 8 semanas & C. Educación \\
\hline 06 & $\begin{array}{l}\text { Designing and Teaching } \\
\text { for Impact in Online } \\
\text { Courses }\end{array}$ & U. Indiana & Canvas Network & abierto & $\begin{array}{l}\text { Diseño de } \\
\text { cursos }\end{array}$ \\
\hline $\mathbf{0 7}$ & $\begin{array}{l}\text { Por los mares de la } \\
\text { traducción económico- } \\
\text { financiera (EN-ES) }\end{array}$ & U. Valladolid & Canvas Network & 7 semanas & $\begin{array}{l}\text { Ciencias } \\
\text { Sociales }\end{array}$ \\
\hline $\mathbf{o 8}$ & $\begin{array}{l}\text { Teaching With } \\
\text { Technology and } \\
\text { Inquiry: An Open } \\
\text { Course For Teachers }\end{array}$ & U. Toronto & $\mathrm{EdX}$ & 8 semanas & C. Educación \\
\hline $\mathbf{0 9}$ & $\begin{array}{l}\text { Launching Innovation } \\
\text { in Schools }\end{array}$ & MIT & $\mathrm{EdX}$ & 6 semanas & C. Educación \\
\hline 10 & $\begin{array}{l}\text { Design Kit: The Course } \\
\text { for Human-Centered } \\
\text { Design }\end{array}$ & $\begin{array}{l}\text { Acumen, } \\
\text { IDEO.org. }\end{array}$ & NovoEd & 7 semanas & $\begin{array}{l}\text { Ciencias } \\
\text { Sociales }\end{array}$ \\
\hline $\mathbf{1 1}$ & $\begin{array}{l}\text { Learning Online: } \\
\text { Learning and } \\
\text { Collaborating }\end{array}$ & U. Leeds & FutureLearn & 2 semanas & $\begin{array}{l}\text { Desarrollo } \\
\text { personal }\end{array}$ \\
\hline
\end{tabular}




\begin{tabular}{|c|c|c|c|c|c|}
\hline Id & Título & Institución & Plataforma & Duración & $\begin{array}{l}\text { Área de } \\
\text { interés }\end{array}$ \\
\hline 12 & $\begin{array}{l}\text { Girls'Education: } \\
\text { Teaching Strategies } \\
\text { That Develop } \\
\text { Confidence, Resilience } \\
\text { and Collaboration }\end{array}$ & $\begin{array}{l}\text { Proyecto } \\
\text { de escuelas } \\
\text { británicas }\end{array}$ & FutureLearn & 4 semanas & $\begin{array}{l}\text { Ciencias } \\
\text { Sociales }\end{array}$ \\
\hline 13 & Gamification & $\begin{array}{l}\text { U. } \\
\text { Pensilvania }\end{array}$ & Coursera & 6 semanas & $\begin{array}{l}\text { Diseño de } \\
\text { cursos }\end{array}$ \\
\hline 14 & Español para viajeros & UNED & OpenMOOC & 4 semanas & Humanidades \\
\hline 15 & $\begin{array}{l}\text { Literacy Teaching } \\
\text { and Learning: Aims, } \\
\text { Approaches and } \\
\text { Pedagogies }\end{array}$ & $\begin{array}{l}\text { U. Illinois } \\
\text { Urbana- } \\
\text { Champaign }\end{array}$ & Coursera & 4 semanas & C. Educación \\
\hline 16 & $\begin{array}{l}\text { Get Interactive: } \\
\text { Practical Teaching with } \\
\text { Technology }\end{array}$ & U. London & Coursera & 3 semanas & C. Educación \\
\hline 17 & $\begin{array}{l}\text { Blended Learning } \\
\text { Essentials: Embedding } \\
\text { Practice }\end{array}$ & $\begin{array}{l}\text { U. Leeds, } \\
\text { UCL }\end{array}$ & FutureLearn & 3 semanas & C. Educación \\
\hline 18 & $\begin{array}{l}\text { LEARNx Deep Learning } \\
\text { through Transformative } \\
\text { Pedagogy }\end{array}$ & $\begin{array}{l}\text { U. } \\
\text { Queensland }\end{array}$ & EdX & 4 semanas & C. Educación \\
\hline 19 & $\begin{array}{l}\text { Leading Change: Go } \\
\text { Beyond Gamification } \\
\text { with Gameful Learning }\end{array}$ & U. Michigan & EdX & 8 semanas & C. Educación \\
\hline 20 & $\begin{array}{l}\text { Gamify Your Canvas } \\
\text { Course with Badgr } \\
\text { Open Badges }\end{array}$ & $\begin{array}{l}\text { Concentric } \\
\text { Sky }\end{array}$ & CanvasNetwork & abierto & $\begin{array}{l}\text { Diseño de } \\
\text { cursos }\end{array}$ \\
\hline
\end{tabular}

Fuente: Elaboración propia (2017)

A continuación, se comentan los resultados de dos de las categorías principales relacionadas con la colaboración, que se refieren a los mecanismos para favorecer la interacción y la comunicación entre participantes (véase tabla 2), y a la posible presencia intencional de aprendizaje colaborativo (véase tabla 3). En el desarrollo del marco, la diferenciación entre ambas categorías se consideró pertinente puesto que, con frecuencia, se observan propuestas de actividades y herramientas en los MOOC que fomentan la interacción con una motivación fundamentalmente social, mientras que en otros casos la meta podría ser lograr una situación de aprendizaje colaborativa, como ocurre con frecuencia en los diseños de aprendizaje para contextos presenciales.

Sin embargo, como se puede apreciar en la tabla 3, se ha observado que en ninguno de los veinte MOOC analizados se desarrollaban tareas en las que ese 
aprendizaje colaborativo fuera el eje central de las actividades propuestas (dicho eje como parte básica del proceso de aprendizaje del curso y que todos los contenidos, dinámicas y actividades giren en torno al desarrollo de ese aprendizaje). Esto no significa que no exista ningún tipo de interacción entre los participantes de los MOOC ya que en las plataformas se incluyen diferentes herramientas sociales (como foros o herramientas para el soporte de revisiones entre pares). De hecho, una vez finalizado el análisis de los veinte MOOC, se observó que las dos herramientas más utilizadas son los foros y las redes sociales.

Tabla 2. Mecanismos para favorecer la interacción entre los participantes (más detalles proporcionados en el material complementario ${ }^{1}$ )

\begin{tabular}{|c|c|c|c|c|c|c|c|c|c|c|c|c|c|c|c|c|c|c|c|c|c|}
\hline \multicolumn{2}{|c|}{ MOOC } & 01 & 02 & 03 & 04 & 05 & 06 & 07 & 08 & 09 & 10 & 11 & 12 & $13 \mid$ & 14 & $15 \mid$ & 16 & $|17|$ & 18 & 19 & 20 \\
\hline \multicolumn{2}{|c|}{ Se crean grupos } & $\mathrm{X}$ & $\mathrm{X}$ & $\mathrm{X}$ & $\mathrm{X}$ & & & $\mathrm{X}$ & $\mathrm{X}$ & $\mathrm{X}$ & $\mathrm{X}$ & & & & & & & & & & \\
\hline \multicolumn{2}{|c|}{ Se crean subgrupos } & $\mathrm{X}$ & & & $\mathrm{X}$ & & & & & & & & & & & & & & & & \\
\hline \multirow{7}{*}{$\begin{array}{l}\text { Herra- } \\
\text { mientas }\end{array}$} & Foros & $\mathrm{X}$ & $\mathrm{X}$ & & $\mathrm{X}$ & & & $\mathrm{X}$ & $\mathrm{X}$ & $\mathrm{X}$ & $\mathrm{X}$ & & & $\mathrm{X}$ & $\mathrm{X}$ & $\mathrm{X}$ & $\mathrm{X}$ & $\mathrm{X}$ & $\mathrm{X}$ & $\mathrm{X}$ & $\mathrm{X}$ \\
\hline & Chats & $\mathrm{X}$ & $\mathrm{X}$ & & & & & & & & & & & & & & & & & & \\
\hline & $\begin{array}{l}\text { Redes } \\
\text { sociales } \\
\end{array}$ & $\mathrm{X}$ & $\mathrm{X}$ & $\mathrm{X}$ & $\mathrm{X}$ & $\mathrm{X}$ & & $\mathrm{X}$ & $\mathrm{X}$ & $\mathrm{X}$ & $\mathrm{X}$ & $\mathrm{X}$ & $\mathrm{X}$ & & $\mathrm{X}$ & $\mathrm{X}$ & $\mathrm{X}$ & $\mathrm{X}$ & & & \\
\hline & Wikis & & & & & & & & $\mathrm{X}$ & & & & & & & & $\mathrm{X}$ & & & & \\
\hline & Blogs & & & & & & & & & & $\mathrm{X}$ & & & & & & & & & & \\
\hline & $\begin{array}{l}\text { Hang- } \\
\text { outs }\end{array}$ & & & & & & & & & & & & & & & & & & & & \\
\hline & Otras & & $\mathrm{X}$ & & $\mathrm{X}$ & & & & $\mathrm{X}$ & $\mathrm{X}$ & $\mathrm{X}$ & $\mathrm{X}$ & $\mathrm{X}$ & & $\mathrm{X}$ & $\mathrm{X}$ & $\mathrm{X}$ & $\mathrm{X}$ & & & \\
\hline
\end{tabular}

Fuente: Elaboración propia (2017) 
Tabla 3. Características del aprendizaje colaborativo que puede darse en cada curso (más detalles proporcionados en el material complementario ${ }^{1}$ )

\begin{tabular}{|c|c|c|c|c|c|c|c|c|c|c|c|c|c|c|c|c|c|c|c|c|c|}
\hline \multicolumn{2}{|c|}{ MOOC } & \multicolumn{2}{|c|}{0102} & \multicolumn{2}{|c|}{0304} & \multicolumn{2}{|c|}{40506} & \multicolumn{4}{|c|}{07080910} & 11 & \multicolumn{3}{|c|}{12131} & \multicolumn{2}{|c|}{1516} & \multicolumn{4}{|c|}{\begin{tabular}{|l|l|l|l|}
17 & 18 & 19 & 20
\end{tabular}} \\
\hline \multirow{6}{*}{ Motivación } & Obligatorio & & & $\mathrm{X}$ & & & & $\mathrm{X}$ & & & & & & & & & & & & & \\
\hline & Espontáneo & & $\mathrm{X}$ & & & & & $\mathrm{X}$ & $\mathrm{X}$ & $\mathrm{X}$ & $\mathrm{X}$ & & & $X$ & $\mathrm{X}$ & & & & $\mathrm{X}$ & $\mathrm{X}$ & $\mathrm{X}$ \\
\hline & $\begin{array}{l}\text { Enriquece el } \\
\text { contenido }\end{array}$ & $\mathrm{X}$ & $\mathrm{X}$ & & $\mathrm{X}$ & $\mathrm{X}$ & & $\mathrm{X}$ & & $\mathrm{X}$ & $\mathrm{X}$ & & & $\mathrm{X}$ & $\mathrm{X}$ & & & & $\mathrm{X}$ & $\mathrm{X}$ & \\
\hline & $\begin{array}{l}\text { Resolver } \\
\text { tareas }\end{array}$ & & & $\mathrm{X}$ & $\mathrm{X}$ & $\mathrm{X}$ & & $\mathrm{X}$ & & $\mathrm{X}$ & $\mathrm{X}$ & $\mathrm{X}$ & & & & & & $\mathrm{X}$ & & & \\
\hline & $\begin{array}{l}\text { Beneficio } \\
\text { indirecto }\end{array}$ & & & $\mathrm{X}$ & & & & & & & & & & & & & & & & & \\
\hline & Desconocido & & & & & & & & & & & & & & & & & & & & \\
\hline \multirow{3}{*}{ Escala } & Gran grupo & $\mathrm{X}$ & $\mathrm{X}$ & $\mathrm{X}$ & $X$ & & & $\mathrm{X}$ & $\mathrm{X}$ & $\mathrm{X}$ & $\mathrm{X}$ & $\mathrm{X}$ & $\mathrm{X}$ & $\mathrm{X}$ & & & & $\mathrm{X}$ & & & \\
\hline & Grupo medio & $\mathrm{X}$ & & & & & & & & $\mathrm{X}$ & & & & & & & & & & & \\
\hline & \begin{tabular}{|l|} 
Pequeño \\
grupo
\end{tabular} & $\mathrm{X}$ & & & & & & & & $\mathrm{X}$ & & & & & & & & & & & \\
\hline \multirow{4}{*}{$\begin{array}{l}\text { Relevancia } \\
\text { de la } \\
\text { colaboración } \\
\text { para el } \\
\text { aprendizaje }\end{array}$} & Fundamental & & & & & & & & & & & & & & & & & & & & \\
\hline & Ocasional & & $\mathrm{X}$ & $\mathrm{X}$ & $X$ & $\mathrm{X}$ & & $\mathrm{X}$ & $\mathrm{X}$ & $\mathrm{X}$ & $\mathrm{X}$ & $\mathrm{X}$ & & & & & & $\mathrm{X}$ & & & \\
\hline & Anecdótico & $\mathrm{X}$ & & & & & & & & & & & & $\mathrm{X}$ & $\mathrm{X}$ & $\mathrm{X}$ & $\mathrm{X}$ & & $\mathrm{X}$ & $\mathrm{X}$ & $\mathrm{X}$ \\
\hline & Inexistente & & & & & & $\mathrm{X}$ & & & & & & & & & & & & & & \\
\hline \multirow{7}{*}{ Recursos } & Foros & X & $\mathrm{X}$ & & $\mathrm{X}$ & & & X & X & X & $X$ & & & & & & & & & & \\
\hline & Chats & & & & $\mathrm{X}$ & & $\mathrm{X}$ & $\mathrm{X}$ & $\mathrm{X}$ & $\mathrm{X}$ & $\mathrm{X}$ & & & & & & & & & & \\
\hline & \begin{tabular}{|l|} 
Redes \\
sociales
\end{tabular} & & & & & & & & & & $\mathrm{X}$ & & & & & & & & & & \\
\hline & Wikis & & & & & & & & $\mathrm{X}$ & & & & & & & & & & & & \\
\hline & $B \log s$ & & & & & $\mathrm{X}$ & & & & & & & & & $\mathrm{X}$ & & & & & & \\
\hline & Hangouts & & & & & & & & $\mathrm{X}$ & $\mathrm{X}$ & $\mathrm{X}$ & & & & & & & & & & \\
\hline & Otras & $\mathrm{X}$ & & $\mathrm{X}$ & $\mathrm{X}$ & $\mathrm{X}$ & & $\mathrm{X}$ & $\mathrm{X}$ & $\mathrm{X}$ & & & & & $\mathrm{X}$ & $\mathrm{X}$ & & $\mathrm{X}$ & & & \\
\hline \multirow{2}{*}{$\begin{array}{l}\text { Evaluación } \\
\text { colaborativa }\end{array}$} & $\begin{array}{l}\text { Por pares } \\
\text { libre }\end{array}$ & & & & & & & & & $\mathrm{X}$ & $\mathrm{X}$ & $\mathrm{X}$ & & & & $\mathrm{X}$ & & $\mathrm{X}$ & & & \\
\hline & $\begin{array}{l}\text { Por pares } \\
\text { con rúbrica }\end{array}$ & $\mathrm{X}$ & $\mathrm{X}$ & $\mathrm{X}$ & $X$ & $X$ & & $\mathrm{X}$ & & $\mathrm{X}$ & $\mathrm{X}$ & & & $X$ & $\mathrm{X}$ & $\mathrm{X}$ & $\mathrm{X}$ & $X$ & & & \\
\hline
\end{tabular}

Fuente: Elaboración propia (2017)

En el caso de los foros, se trata de espacios normalmente integrados dentro de los propios cursos, cuya función varía entre fomentar las relaciones sociales entre usuarios, expandir la información aportada en los bloques temáticos, resolver dudas o crear temas de debate. Algunos foros tienen una duración longitudinal a lo largo del curso (normalmente aquellos asociados a más generales, como sociales o de 
apoyo técnico) mientras que otros tienen un uso acotado en el tiempo (normalmente asociados a un módulo de contenidos).

Por su parte, las redes sociales más utilizadas son Facebook y Twitter. En Facebook se tiende a crear grupos de discusión, en el que los usuarios del MOOC cuentan con un espacio más a través del cual poder interrelacionarse o hablar sobre determinadas cuestiones del curso. Estos grupos de Facebook sirven como extensiones de los foros incluidos en las plataformas, aprovechando la familiaridad de muchos usuarios con esta plataforma. El uso de determinados hashtags es el modo en el que Twitter suele utilizarse como intermediario social entre los docentes del curso y sus usuarios. Los hashtags permiten crear líneas de conversación sobre temas concretos que proponen los docentes.

En este sentido, en la mayoría de los MOOC se plantean las actividades en las que el alumnado interactúa en gran grupo debido por una parte a las limitaciones de las plataformas actuales a la hora de formar grupos pequeños y por otra, por el alto grado de abandono (Sanz-Martínez, Muñoz-Cristóbal, Bote-Lorenzo, Martínez-Monés y Dimitriadis, 2017). Sin embargo, en el MOOCo7 para propiciar la interacción se proponen actividades en grupo pequeño formando cohortes de seis alumnos basados en su actividad pasada (páginas vistas, tareas enviadas, mensajes en foros) (Sanz-Martínez et al., 2017). Además en este mismo curso, se llevó a cabo una actividad obligatoria colaborativa (extracción terminológica) y que también se realizó en pequeños grupos mediante un foro grupal previamente habilitado y la interfaz de grupos que ofrece la plataforma que aloja dicho curso (Canvas Network).

En la mayoría de los MOOC analizados, la interacción social a través de los medios anteriormente descritos desempeña un papel relevante dado que se hace alusión a ella a lo largo de todo el curso. Se recuerda constantemente a los usuarios que utilicen los foros y las redes sociales, e incluso (aunque sin ser obligatorio) la experiencia de aprendizaje se expande a través de estos medios ya que se pide que se reflexione y se debata con el resto de usuarios sobre las actividades propuestas. Así, a través de estos medios termina por crearse un proceso de aprendizaje colaborativo espontáneo. Tal y como se define esta categoría en el marco de análisis, es un tipo de colaboración que "surge de manera imprevista por iniciativa propia por los participantes del curso al compartir experiencias a través de las herramientas de comunicación presentes en los cursos" (García-Sastre et al., 2017, p. 65). Dicha interacción social ayudaba a enriquecer el contenido propuesto y se convertía al mismo tiempo en canalizador de los conocimientos teóricos y en lugar de encuentro para los participantes, que al interrelacionarse entre sí, desarrollaban procesos de aprendizaje colaborativo de manera indirecta. Por el contrario, ninguno de los MOOC analizados presenta un diseño que permitiera afirmar que el aprendizaje colaborativo es columna vertebral del proceso de enseñanza. Sin embargo, las cuestiones anteriores permiten afirmar que el aprendizaje colaborativo se produce de manera "ocasional".

Como elemento diferenciador, el MOOCo8, ofrecido por la Universidad de Toronto en la plataforma EdX, emplea una wiki como herramienta para la interacción 
social y la colaboración. En el extremo opuesto, en el MOOCo6 (Universidad de Indiana) no se pone a disposición del usuario ningún tipo de herramienta ni para la interacción social ni la colaboración (nótese la paradoja de que ambos cursos son sobre formación con tecnología, pero uno enseña e intenta practicar una pedagogía activa, incluso a nivel masivo, mientras que el otro sigue la aproximación instruccional dominante en los MOOC).

Finalmente, otro elemento en el que se observa la presencia de la colaboración es la evaluación. Como también es susceptible de ser colaborativa, fue analizada en los diferentes MOOC para comprobar qué planteamientos se seguían. El único tipo de evaluación que puede considerarse colaborativa, y que sí ha sido utilizada en la mayoría de los MOOC, es el peer assessment, casi siempre apoyado por rúbrica. El marco de análisis también propone estudiar si se producen otras variantes de evaluación colaborativa, como por ejemplo el portafolio, pero en los MOOC analizados solo se ha detectado el uso del mencionado peer assessment (por este motivo, en la tabla 3 se han omitido otros mecanismos de evaluación colaborativa).

$\mathrm{El}$ otro foco de atención de este estudio es la gamificación. Como se resume en la tabla 4, se ha encontrado presencia de la gamificación en algunos de los cursos analizados, aunque no es muy frecuente.

Tabla 4. Características de la gamificación en aquellos cursos en los que se utiliza (más detalles proporcionados en el material complementario ${ }^{1}$ )

\begin{tabular}{|c|c|c|c|c|c|c|c|}
\hline \multicolumn{2}{|c|}{ MOOC } & 01 & 05 & 07 & 14 & 16 & 20 \\
\hline \multirow{5}{*}{ Finalidad } & Motivación & $\mathrm{X}$ & & $\mathrm{X}$ & & & $\mathrm{X}$ \\
\hline & Fomentar comportamientos & & & $\mathrm{X}$ & $\mathrm{X}$ & & \\
\hline & Mejorar aprendizaje & $\mathrm{X}$ & & $\mathrm{X}$ & & & \\
\hline & Otros & & & & & $\mathrm{X}$ & \\
\hline & Desconocido & & $\mathrm{X}$ & & & & \\
\hline \multirow{13}{*}{ Mecánicas y dinámicas } & Puntos & & & & & & \\
\hline & Niveles & & & & & & \\
\hline & Leaderboard / Rankings & & & $\mathrm{X}$ & & & \\
\hline & Gifts & & & & & & \\
\hline & Medallas & $\mathrm{X}$ & $\mathrm{X}$ & $\mathrm{X}$ & $\mathrm{X}$ & $\mathrm{X}$ & \\
\hline & Temporizadores & & & & & & \\
\hline & Vidas & & & & & & \\
\hline & Objetos virtuales canjeables & & & & & & \\
\hline & Narrativa & & & & & & \\
\hline & Misiones & & & & & & \\
\hline & Competición & & & & & & \\
\hline & Colaboración & & & & & & \\
\hline & Desbloqueo de contenidos & & & & & & \\
\hline
\end{tabular}




\begin{tabular}{|l|l|c|c|c|c|c|c|}
\hline \multicolumn{2}{|l|}{ MOOC } & $\mathbf{0 1}$ & $\mathbf{0 5}$ & $\mathbf{0 7}$ & $\mathbf{1 4}$ & $\mathbf{1 6}$ & $\mathbf{2 0}$ \\
\hline \multirow{5}{*}{ Tipo } & Elementos sociales & & & & & & $\mathrm{X}$ \\
\hline & Otros & & & & & & \\
\hline \multirow{3}{*}{ Herramientas } & Individual & $\mathrm{X}$ & $\mathrm{X}$ & $\mathrm{X}$ & $\mathrm{X}$ & $\mathrm{X}$ & $\mathrm{X}$ \\
\hline & Colectiva & & & & & & \\
\hline & Las de la plataforma MOOC & & & & & & \\
\hline & Externas & & & & & & \\
\hline & Ambas & & & & & & \\
\hline
\end{tabular}

Fuente: Elaboración propia (2017)

Se observó la singularidad de uno de los cursos, el MOOCo7 en el que las medallas son otorgadas como recompensa a los estudiantes por sus determinadas acciones, además de ser motivación para completar los módulos e interactuar en el foro con otros estudiantes o instructores y con los miembros de su grupo (Ortega-Arranz, Muñoz-Cristóbal, Martínez-Monés, Bote-Lorenzo y Asensio-Pérez, 2017b). En este y en algunos otros cursos se conceden medallas en acciones no obligatorias a medida que se va avanzando en el curso. En el MOOC14, "Español para viajeros" (Sedano, 2017), también se otorgan medallas por completar actividades de revisión entre pares y por dar y recibir comentarios en los foros. Igualmente, en una actividad del MOOC16 se aprecia que los participantes, una vez conseguidas las medallas, pueden mostrarlas en otras actividades del MOOC como prueba de sus logros anteriores. Otro mecanismo de gamificación usado en el MOOCo7 es la incorporación de un leaderboard (ranking) que compara las medallas ganadas por los distintos estudiantes.

Asimismo, solo el MOOCo7, "Por los mares..." hace una primera aproximación a la hora de otorgar recompensas en actividades que involucran pequeños grupos, aunque las acciones siguen estando limitadas a la participación en los foros de los grupos y a los envíos de tareas grupales (Ortega-Arranz et al., 2017b). Es decir, la gamificación apenas se está usando en MOOC de manera explícita para fomentar diseños que garanticen la colaboración más allá de la participación en foros y el envío de revisiones entre pares.

Por último, en lo que concierne a la tecnología de apoyo a la gamificación, se observa que con frecuencia se usan herramientas de terceros para gestionar los elementos de juegos, a pesar de que las acciones que se gamifican (las que permiten obtener las medallas) son las que ocurren dentro de las plataformas MOOC. En el caso concreto del MOOC14 se usa Open Badges, (estándar que permite la inclusión de meta-información junto a la recompensa, permitiendo su exportación a otras plataformas o sitios web, y la adición de información, como por ejemplo el motivo por el que se obtuvo, etc.) a través de la herramienta Karma en la plataforma de OpenMOOC. En otro de los cursos, en el MOOCo7, la herramienta de terceros utilizada es Badgr que permite recompensar con medallas las acciones realizadas por 
los estudiantes dentro de la propia plataforma donde está alojado el curso, Canvas Network. La conexión entre la plataforma y la herramienta se produce mediante el estándar LTI (Learning Tools Interoperability) ${ }^{10}$ permitiendo el intercambio de información y la acreditación y almacenaje de las medallas. También, en este MOOC, se pretende recompensar a los estudiantes por añadir términos a un glosario colaborativo implementado con una herramienta externa (Google Form y Spreadsheet). Sin embargo, debido a la realización de la actividad en una herramienta externa a la plataforma MOOC, se tuvo que pedir a los estudiantes que volvieran a incluir esos términos en una actividad configurada con las herramientas de Canvas para poder otorgarles las recompensas (Ortega-Arranz et al., 2017b).

\section{DISCUSIÓN Y CONCLUSIONES}

Las evidencias obtenidas tras analizar los veinte MOOC seleccionados vienen a mostrar que el formato actual de estos cursos ha ido avanzando, aunque muy tímidamente, hacia la incorporación de pedagogías activas. Por una parte, es frecuente encontrar una serie de actividades cuyo objetivo es potenciar la interacción social entre usuarios, lo que puede fomentar una sensación de comunidad y favorecer la retención de los estudiantes y, en algunas ocasiones, generar colaboración espontánea. Además, estas actividades se apoyan casi exclusivamente en foros o redes sociales como centros de debate y opinión, y no se incluyen otras herramientas que permitan la creación de resultados de aprendizaje colaborativos, lo cual enriquecería mucho los procesos de enseñanza propuestos en los MOOC. Plantear de antemano actividades en las que los usuarios deban trabajar de forma colaborativa para llevar a cabo una tarea concreta sería una opción deseable que ayudaría a dar un paso más allá en la interacción y que permitiría hablar de aprendizaje colaborativo alcanzado de una manera intencionada.

Por otra parte, la escala puede ser percibida por los diseñadores instruccionales como un problema para gestionar trabajos grupales. En la gran mayoría de los MOOC las actividades en las que se espera que los alumnos interactúen se plantean para el gran grupo, lo que puede hacer que la creación de vínculos de trabajo entre los usuarios se difumine y pierda su potencial. La creación de subgrupos es algo que debería considerarse más detenidamente, puesto que ayuda a centrar el interés y delimitar las relaciones entre los participantes. Eso sí, con altas tasas de abandono, se necesitan mecanismos efectivos de asignación y reasignación dinámica de alumnos a los grupos, para garantizar que un alumno siempre tenga otros con los que interactuar (Sanz-Martínez et al., 2017).

En lo que respecta a la gamificación, los MOOC analizados arrojan cierta evidencia de su uso como elemento motivador para mantener la implicación del alumno y evitar su abandono. En ocasiones, también se utiliza para fomentar la interacción en el gran grupo premiando, por ejemplo, las participaciones en un foro. Sin embargo, existe un gran potencial en el uso de la gamificación para recompensar 
acciones logradas colectivamente en pequeño grupo y así fomentar interacciones significativas de colaboración, pero apenas se ha observado una evidencia de este uso.

Un aspecto adicional que puede limitar la puesta en práctica de actividades colaborativas y gamificadas es el apoyo dado por las plataformas tecnológicas en las que se crean y distribuyen los MOOC. Aunque las dificultades que la tecnología impone a los diseñadores instruccionales son más difíciles de apreciar en un análisis a posteriori de los cursos, sí se observa por ejemplo la tendencia a la utilización de herramientas de gamificación de terceros. Esto puede perseguir el objetivo de exportar medallas y así dar mayor visibilidad a los resultados del MOOC, pero también muestra la limitación o inexistencia de herramientas en las propias plataformas. En el caso de la colaboración, también se detecta que las herramientas disponibles por defecto suelen limitarse a foros de discusión, y que se recurre a redes sociales o incluso a wikis externas para complementarlas. Otra dificultad presente en muchas plataformas es que ni siquiera existe el concepto de "grupo" (aunque en este sentido es relevante observar cómo los proveedores de tecnología han detectado esta necesidad, y por ejemplo EdX ha introducido el concepto mediante las denominadas "cohortes").

En resumen, se aprecia una mayor preocupación por la interacción social, el uso de gamificación para mejorar la implicación y, en ocasiones, se detecta que ocurren actividades de aprendizaje colaborativo espontáneo, pero todavía no se encuentran cursos en los que estas situaciones sean perseguidas desde el diseño de manera explícita.

Es necesario por lo tanto investigar en mejorar las tecnologías para apoyar el diseño y puesta en práctica de actividades colaborativas en los cursos masivos. En esta línea, el ILDE (Integrated Learning Design Environment, Entorno Integrado de Diseño de Aprendizaje) (Hernández-Leo, Asensio-Pérez, Derntl, Prieto y Chacón, 2014) es una plataforma que permite formalizar computacionalmente diseños de aprendizaje, aportando al profesor consejos y buenas prácticas para el uso de la colaboración, y posteriormente desplegar estos diseños en plataformas de aprendizaje (como Moodle). Se ha usado para desplegar diseños de varios cientos de estudiantes en Moodle, pero todavía no ofrece soporte para desplegar cursos en plataformas MOOC como Canvas o EdX, aunque es parte de investigaciones en curso. Otras herramientas de apoyo al profesor para diseñar MOOC, como el marco propuesto por Alario-Hoyos, Pérez-Sanagustín, Cormier y Delgado-Kloos (2014) y su plataforma asociada ${ }^{11}$ asesoran al diseñador en la conceptualización de un curso masivo, pero no tienen énfasis en la colaboración. Más allá del diseño y despliegue, existen algunas tecnologías que se han propuesto para facilitar la colaboración de los alumnos en alta escala. Por ejemplo, PyramidApp (Manathunga y HernándezLeo, 2016) permite que los alumnos vayan realizando la misma actividad en grupos sucesivamente más grandes, hasta alcanzar un consenso de gran grupo, usando esta aplicación desde su teléfono móvil, pero viendo sus resultados reflejados en 
la plataforma de aprendizaje. Los agentes conversacionales automáticos han sido probados con éxito para fomentar la colaboración (Tegos, Demetriadis y Karakostas, 2015), y pueden desempeñar el papel de dinamizadores del debate en lugar del profesor cuando la escala es masiva.

Desde un punto de vista más pedagógico, es necesario poner realmente en práctica diseños colaborativos en contextos masivos para analizar los problemas encontrados por los educadores. También sería muy deseable analizar las intenciones pedagógicas de los diseñadores instruccionales que crean estos cursos, participando en el codiseño, en lugar de simplemente analizar los cursos a posteriori. Eso permitiría formular de manera más precisa las dificultades percibidas, derivadas de la tecnología, la escala, el perfil de los alumnos o cualquier otra causa, para llevar a cabo situaciones de aprendizaje colaborativo en cursos online masivos y abiertos.

\section{APOYOS Y AGRADECIMIENTOS}

Esta investigación se ha llevado a cabo gracias al apoyo parcial del proyecto regional de la Junta de Castilla y León (España) (VAo82U16) y de dos proyectos nacionales del Ministerio de Economía y Competitividad (España) (TIN2014-53199$\mathrm{C}_{3}-2-\mathrm{R}$ y TIN2017-85179-C3-2-R); los autores agradecen el apoyo de las personas que forman parte del grupo de investigación GSIC/EMIC.

\section{NOTAS}

1. Material complementario. Marco de análisis de categorías MOOC

2. https://owncloud.gsic.uva.es/index.php/s/eoXQqmQx5wKkPgm

3. Canvas Network https://www.canvas.net

4. ECOLearning (OpenMOOC) https://ecolearning.eu

5. Coursera https://www.coursera.org

6. FutureLearn https://www.futurelearn.com

7. EdX https://www.edx.org/es

8. NovoEd https://novoed.com

9. MiríadaX https://miriadax.net/hom

10. Class Central. Motor de búsqueda de MOOC http://www.class-central.com

11. LTI (Learning Tools Interoperability) http://www.imsglobal.org/activity/learning tools-interoperability

12. MOOC Canvas. Universidad Carlos III. Madrid. https://www.it.uc3m.es/calario/ MOOCCanvas,

\section{REFERENCIAS BIBLIOGRÁFICAS}

Alario-Hoyos, C., Pérez-Sanagustín, M., Delgado-Kloos, C., Parada-Gélvez, H. A., y Muñoz-Organero, M. (2014). Delving into participants' profiles and use of social tools in MOOCs. IEEE Transactions on Learning Technologies, 7(3), 260- 
266. doi: https://doi.org/10.1109 TLT.2014.2311807

Alario-Hoyos, C., Pérez-Sanagustín, M., Cormier, D., y Delgado-Kloos, C. (2014). Proposal for a conceptual framework for educators to describe and design MOOCs. Journal of Universal Computer Science, 2O(1), 6-23. Recuperado de http://goo. gl/oLpoiR

Balula, A. (2015). The promotion of digital inclusion through MOOC design and use: a literature review. Indagatio Didactica, Z(1), 145-164. Recuperado de http://goo. gl/Oq1jNy

Bonwell, C. C., y Eison, J. A. (1991). Active Learning: Creating Excitement in the Classroom. ASHE-ERIC Higher Education Report No.1. Washington, D.C. Recuperado de http://goo.gl/ojPNAi

Claros, I., Echeverría, L., Garmendía, A., y Cobos, R. (2014). Towards a Collaborative Pedagogical Model in MOOCs. In Global Engineering Education Conference (EDUCON), IEEE (pp. 905-911). Estambul, Turquía. doi: https://doi. org/10.1109/EDUCON.2014.6826204

Collazos, C. A., González, C. S., y García, R. (2014).ComputerSupported Collaborative MOOCs: CSCM. In Proceedings of the 2014 Workshop on Interaction Design in Educational Environments (pp. 28-32). ACM. Albacete, España. doi: https://doi. org/10.1145/2643604.2643629

De Sousa Borges, S., Durelli, V., Reis, H., y Isotani, S. (2014), A systematic mapping on gamification applied to education. In Proceedings of the $29^{\text {th }}$ Annual ACM Symposium on Applied Computing, (pp. 216-222). ACM. Gyeongju, Korea. doi: https://doi. org/10.1145/2554850.2554956

Deterding, S., Dixon, D., Khaled, R., y Nacke, L. (2011). From game design elements to gamefulness: defining "gamification". In Proceedings of the $15^{\text {th }}$ International Academic MindTrek Conference: Envisioning Future
Media Environments (pp. 9-15). ACM. Tampere, Finlandia. doi: https://doi. org/10.1145/2181037.2181040

Dichev, C., y Dicheva, D. (2017). Gamifying education: what is known, what is believed and what remains uncertain: a critical review. International Journal of Educational Technology in Higher Education, 14(9), 1-36. doi: https://doi. org/10.1186/s41239-017-0042-5

Dicheva, D., Dichev, C., Agre, G., y Angelova, G. (2015). Gamification in Education: A Systematic Mapping Study. Educational Technology \& Society, 18(3), 75-88. Recuperado de http://goo.gl/FAkQaz

Dillenbourg, P. (1999). What do you mean by collaborative learning? In P. Dillenbourg (Ed.), Collaborative-learning: Cognitive and Computational Approaches (1-19). Oxford: Elsevier.

Dillenbourg, P., Fox, A., Kirchner, C., Mitchell, J., y Wirsing, M. (2014). Massive Open Online Courses: Current state and perspectives (Dagstuhl Perspectives workshop 14112). Dagstuhl Manifestos, 4(1), 1-27. Schloss Dagstuhl-LeibnizZentrum fuer Informatik. Recuperado de http://goo.gl/zwVyPa

Ferguson, R., y Sharples, M. (2014). Innovative Pedagogy at Massive Scale: Teaching and Learning in MOOCs. In C. Rensing, S. de Freitas, T. Ley, y P. J. Muñoz-Merino (Eds.), Open Learning and Teaching in Educational Communities. EC-TEL 2014. Lecture Notes in Computer Science, vol 8719 (pp. 98-111). Springer, Cham. Graz, Austria. doi: https://doi. org/10.1007/978-3-319-11200-8 8

Fidalgo, A., Sein-Echaluce, M. L., y GarcíaPeñalvo, F. J. (2013). MOOC cooperativo. Una integración entre cMOOC y xMOOC. En Á. Fidalgo, y M. L. SeinEchaluce, (Eds.), Actas del II Congreso Internacional sobre Aprendizaje, Innovación y Competitividad, CINAIC (pp. 481-486). Madrid: Fundación General de la Universidad Politécnica de 
Madrid. Recuperado de http://goo.gl oxA06I

García-Sastre, S., Idrissi-Cao, M., OrtegaArranz, A., Muñoz-Cristóbal, J. A., y Gómez-Sánchez, E. (2017). Marco para el Análisis de la Colaboración y la Gamificación en MOOC. Actas de la Fifth European MOOCs Stakeholders Summit, eMOOCs 2017 (pp. 62-71) Madrid, España. Recuperado de http://goo.gl/ bAx7Gn

Gütl, C., Rizzardini, R. H., Chang, V., y Morales, M. (2014). Attrition in MOOC: lessons learned from drop-out students. In L. Uden, J. Sinclair, Y. H. Tao y D. Liberona (Eds.), In Proceedings of the $3^{\text {rd }}$ International Workshop on Learning Technology for Education in Cloud: MOOC and Big Data (pp. 3748). Springer, Cham. doi: https://doi. org/10.1007/978-3-319-10671-7 4

Hamari, J., Koivisto, J., y Sarsa, H. (2014). Does gamification work?-a literature review of empirical studies on gamification. In Proceedings of the $47^{\text {th }}$ Hawaii International Conference on System Sciences, HICSS (pp. 30253034). IEEE. Hawaii. doi: https://doi. org/10.1109/HICSS.2014.377

Hansch, A., Newman, C., y Schildhauer, T. (2015). Fostering Engagement with Gamification: Review of Current Practices on Online Learning Platforms. HIIG Discussion Paper Series. doi: https://doi. org/10.2139/ssrn.2694736

Hernández-Leo, D., Asensio-Pérez, J. I., Derntl, M., Prieto, L. P., y Chacón, J. (2014). ILDE: Community Environment for Conceptualizing, Authoring and Deploying Learning Activities. In Proceedings of the $9^{\text {th }}$ European Conference on Technology Enhanced Learning, EC-TEL 2014 (pp. 490-493). Graz, Austria. Recuperado de http://goo. gl/My2Twd

Jordan, K. (2014). Initial trends in enrolment and completion of massive open online courses. The International Review of Research in Open and Distributed Learning, 15(1), 133-160. doi:https://doi. org/10.19173/irrodl.v15i1.1651

Manathunga, K., y Hernández-Leo, D. (2015). Has Research on Collaborative Learning Technologies Addressed Massiveness? A Literature Review. Educational Technologies \& Societu, 18(4), 357-370. Recuperado de http://goo.gl/4MJulE

Manathunga, K., y Hernández-Leo, D. (2016). PyramidApp: scalable method enabling collaboration in the classroom. In K. Verbert, M. Sharples, y T. Klobučar (Eds.). Adaptive and adaptable learning: In Proceedings of the $11^{\text {th }}$ European Conference on Technology Enhanced Learning, EC-TEL 2016 (pp.422-427). Lyon, France. Heidelberg: Springer (LNCS, no. 9891). doi: https://doi. org/10.1007/978-3-319-45153-4 37

Margaryan, A., Bianco, M., y Littlejohn, A. (2015). Instruccional Quality of Massive Open Online Courses (MOOCs). Computers \& Education, 80, 7783. doi: https://doi.org/10.1016/j. compedu.2014.08.005

Muñoz, G. R., Collazos, C. A., y González, C. S. (2016). Propuesta para la gamificación de actividades educativas colaborativas en CSCM. Campus Virtuales, 5(2), 18-28. Recuperado de http://goo.gl/ZwZCB3

Ortega-Arranz, A., Muñoz-Cristóbal, J. A., Martínez-Monés, A., Bote-Lorenzo, M. L., y Asensio-Pérez, J. I. (2017a). How Gamification is Being Implemented in MOOCs? A Systematic Literature Review. In Proceedings of the $13^{\text {th }}$ European Conference on Technology Enhanced Learning (pp. 441-447), Tallin, Estonia. Springer, Cham. doi: https://doi. org/10.1007/978-3-319-66610-5 40

Ortega-Arranz, A., Muñoz-Cristóbal, J. A., Martínez-Monés, A., Bote-Lorenzo, M. L., y Asensio-Pérez, J. I. (2017b). Gamifying Collaborative Activities in MOOCs. In Proceedings of the $5^{\text {th }}$ European MOOCs 
Stakeholders Summit (Work In Progress), eMOOCs 2017 (pp. 28-33), Ceur. Madrid, España. Recuperado de http://goo.gl/ UmwaWS

Poy, R., y Gonzales-Aguilar, A. (2015). Boom-bust of MOOC platforms: Crisis of an eLearning model? International Journal of Education and Research, 3(1), 405-410. Recuperado de http://goo.gl/ Z4TBlW

Ramírez-Donoso, L., Pérez-Sanagustín, M., Neyem, A., y Rojas-Riethmuller, J. S. (2015). Promoviendo la Colaboración Efectiva en MOOCs a través de Aplicaciones Móviles. In Proceedings of the IEEE CHILECON. Santiago de Chile. Recuperado de http://goo.gl/LZX7Ni

Sanz-Martínez, L., Muñoz-Cristóbal, J. A., Bote-Lorenzo, M. L., MartínezMonés, A., y Dimitriadis, Y. (2017). Toward Criteria-Based Automatic Group Formation in MOOCs. In Proceedings of the $5^{\text {th }}$ European MOOCs Stakeholders Summit (Work In Progress), eMOOCs 2017 (pp. 83-88), Ceur. Madrid, España. Recuperado de http://goo.gl/DyfX40

Sedano, B. (2017). La atención a las necesidades y demandas específicas del alumnado en un mundo globalizado: el caso de un MOOC de español para viajar. RIED: Revista Iberoamericana de Educación a Distancia 2O(1), 161-182. doi: https://doi.org/10.5944/ried.20.1.16692
Shah, D. (December 21, 2015). By The Numbers: MOOC in 2015. How has the MOOC space grown this year? Get the facts, figures, and pie charts, Class Central. Recuperado de http://goo.gl/fC2ego

Shah, D. (December 25, 2016). By The Numbers: MOOCs in 2016. How has the MOOC space grown this year? Get the facts, figures, and pie charts, Class Central. Recuperado de http://goo.gl fsupug

Tegos, S., Demetriadis, S., y Karakostas, A. (2015). Promoting academically productive talk with conversational agent interventions in collaborative learning settings. Computers \& Education, 87, 309-325. doi: https://doi.org/10.1016/j. compedu.2015.07.014

Zheng, S., Rosson, M. B., Shih, P. C., y Carroll, J. M. (2015). Designing MOOCs as Interactive Places for Collaborative Learning. In Proceedings of the $2^{\text {nd }}$ ACM Conference on Learning@Scale (pp. 343-346). ACM, Vancouver, BC, Canadá. doi: https://doi. org/10.1145/2724660.2728689

Zeng, H. (2016). Gamification Study Scenario Design in MOOCs. In Proceedings of the $3^{\text {rd }}$ International Conference on Information and Communication Technology for Education (ICTE), DEStech Transactions on Computer Science and Engineering, Toronto, Canadá. Recuperado de http:// goo.gl/OHVEjI

\section{PERFIL ACADÉMICO Y PROFESIONAL DE LOS AUTORES}

Sara García-Sastre es profesora del Departamento de Pedagogía y Doctora por la Universidad de Valladolid (2015). Es miembro del grupo de investigación GSIC-EMIC (Grupo de Sistemas Inteligentes y Cooperativos en Educación, Medios, Infraestructura y Cultura), y del Grupo Transdisciplinar de Investigación en Educación (CETIE-UVa) de la Universidad de Valladolid. Sus intereses en investigación se centran en las TIC, en la Tecnología de Aprendizaje Mejorado (TEL) y en el uso del aprendizaje colaborativo y la gamificación en MOOC.

E-mail: saragar@pdg.uva.es 
Miriam Idrissi-Cao obtuvo su Grado en Educación Primaria (2015) y el Máster de Investigación aplicada a la Educación (2016) por la Universidad de Valladolid. Actualmente realiza su tesis doctoral en el Departamento de Pedagogía en la Facultad de Educación y Trabajo Social de Valladolid. Sus intereses de investigación versan en torno a los procesos de enseñanza del inglés como lengua extranjera y el empleo de las TIC como elemento transformador en la escuela.

E-mail: misicao.miriam@gmail.com

\section{DIRECCIÓN DE LAS AUTORAS}

Facultad de Educación y Trabajo Social

Universidad de Valladolid

Departamento de Pedagogía

Paseo de Belén, 1

Valladolid (España)

Alejandro Ortega-Arranz recibió su Grado y Máster en Ingeniería de Telecomunicaciones por la Universidad de Valladolid (España) en 2014 y 2015 respectivamente. Actualmente, es estudiante de doctorado en el grupo deinvestigación GSIC-EMIC (Grupo de Sistemas Inteligentes y Cooperativos en Educación, Medios, Infraestructura y Cultura) en la misma Universidad. Sus principales intereses de investigación incluyen los MOOC, el aprendizaje basado en juegos, la gamificación y las tecnologías que dan soporte a estas prácticas.

E-mail: alex@gsic.uva.es

Eduardo Gómez-Sánchez es Ingeniero de Telecomunicación (1996) y Doctor por la Universidad de Valladolid (2001). Es miembro fundador del Grupo de Sistemas Inteligentes y Cooperativos (GSIC), Unidad de Investigación Consolidada que investiga en el ámbito del aprendizaje apoyado por ordenador. Sus intereses de investigación particulares son la aplicación de sistemas distribuidos al aprendizaje ubicuo, las analíticas de aprendizaje y el impacto, tanto tecnológico como pedagógico, de la escala.

E-mail:edugom@tel.uva.es

\section{DIRECCIÓN DE LOS AUTORES}

Escuela Técnica Superior de Ingenieros de Telecomunicación

Universidad de Valladolid

Departamento de Teoría de la Señal y Comunicaciones e Ingeniería Telemática.

Paseo de Belén, 15

Valladolid (España) 
Fecha de recepción del artículo: 15/11/2017

Fecha de aceptación del artículo: 04/01/2018

\section{Como citar este artículo:}

García Sastre, S., Idrissi-Cao, M., Ortega Arranz, A., Gómez Sánchez, E. (2018). Uso de la colaboración y la gamificación en MOOC: un análisis exploratorio. RIED. Revista Iberoamericana de Educación a Distancia, 21(2), pp. 263-283. doi: http:// dx.doi.org/10.5944/ried.21.2.20410 